\title{
My virtual self: the role of movement in children's sense of embodiment
}

\author{
Hayley Dewe*, Janna M. Gottwald*, Laura-Ashleigh Bird, Harry Brenton, Marco Gillies, \& Dorothy \\ Cowie
}

\begin{abstract}
There are vast potential applications for children's entertainment and education with modern virtual reality (VR) experiences, yet we know very little about how the movement or form of such a virtual body can influence children's feelings of control (agency) or the sensation that they own the virtual body (ownership). In two experiments, we gave a total of 197 children aged 4-14 years a virtual hand which moved synchronously or asynchronously with their own movements and had them interact with a VR environment. We found that movement synchrony influenced feelings of control and ownership at all ages. In Experiment 1 only, participants additionally felt haptic feedback either congruently, delayed or not at all - this did not influence feelings of control or ownership. In Experiment 2 only, participants used either a virtual hand or non-human virtual block. Participants embodied both forms to some degree, provided visuomotor signals were synchronous (as indicated by ownership, agency, and location ratings). Yet, only the hand in the synchronous movement condition was described as feeling like part of the body, rather than like a tool (e.g., a mouse or controller). Collectively, these findings highlight the overall dominance of visuomotor synchrony for children's own-body representation; that children can embody non-human forms to some degree; and that embodiment is also somewhat constrained by prior expectations of body form.
\end{abstract}

Index Terms - Agency, Body Ownership, Embodiment, Synchronous integration, Psychology, User Interaction, Virtual Reality

\section{INTRODUCTION}

$\mathrm{O}_{\mathrm{e}}^{\mathrm{N}}$ NE'S sense of embodiment is a core part of human existence and covers the sensation of ownership over one's body, a feeling of being in control of it (agency), and an awareness of its position and location [1]-[3]. We feel embodied during daily interactions with the real world, but also in virtual reality (VR). Embodiment for a virtual body (or 'virtual self') is one of the key illusions that make up the experience of VR [4]. Research has shown that sensations of embodiment for a virtual body arise with the integration of congruent (i.e., synchronous) multisensory information, such as visual, motor and proprioceptive signals. These signals are compared against internal prior knowledge about the body, such as its expected appearance or movement ability [5]-[8] to determine whether the body is one's own. While the processes giving rise to a sense of embodiment are often explored in adults, it is also important to understand these systems in children. At this age, the body (and its internal representations) are still developing, and the role of multisensory processes for embodiment develops significantly across childhood [9]. Yet, little is known about the factors that constrain embodiment in young children, or the degree of acceptance of a virtual body.

- H. Dewe is at Durham University, UK, hayley.1.dewe@durham.ac.uk

- J.M. Gottwald is at Uppsala University, Sweden, janna.gottwald@psyk.uu.se

- L-A. Bird is at University College London, UK, 1.bird@ucl.ac.uk

- H. Brenton is at Bespoke VR, London,UK, harry@bespokevr.com

- M. Gillies is at Goldsmiths, University of London, UK, M.Gillies@gold.ac.uk

- D. Cowie is at Durham University, UK, dorothy.cowie@durham.ac.uk
VR offers an exciting opportunity to explore the underlying processes and development of self and body representation. It provides a practically limitless and dynamic experience that allows researchers to manipulate the effects, strength and constraints of embodiment of a virtual body. It is not yet understood how children interact within VR [10], [11], and the extent of ownership or agency they feel for virtual bodies. This is problematic given its wide usage [12] and the powerful effects of VR on attitudes and behaviours observed in adults [13]-[15]. It is therefore important to understand the nature of VR embodiment in children.

We present two independent experiments that explored the effects and constraints of embodiment for virtual hands in children. We focused on the congruency of movement, and the role it plays in body representation and children's tendency to accept or embody a virtual hand as their own. In addition, we explored whether additional haptic feedback (tactile stimulation) would increase embodiment for a virtual hand (Exp. 1), and whether a virtual hand of a different virtual appearance could be embodied (Exp. 2). This was achieved using a VR bubble popping task, using an Oculus Rift headset. The task was designed to elicit regular movements that children of all ages could complete using their hand. It was appealing to children of all ages: the bubble changed regularly from transparent to a bright blue colour, providing an easy cue to move on to the next bubble, and the game itself was situated within a fun and vibrant funfair virtual environment. This was similar to previous experiments with adults that used balloon-popping tasks with moving virtual avatars [16]; as well as using virtual or real-life balloon stimuli for the 
embodiment of non-human objects [17], attentional processes [18], [19] or tool use [20].

In these studies, we focused on the experience of hands. They are likely the most viewed body part in current VR experiences, form a crucial interface between the user and the virtual world and go back to some of the earliest experiments in immersive VR [21]. Standard development kits for consumer VR platforms such as the Oculus Rift include the ability to display hands at the position of the controllers, which is a common feature in many experiences. We know that they feature prominently in the visual scene even for infants [22], and knowledge so far suggests that embodiment of hands may follow a specific developmental time course separate to the embodiment of a whole body [9], [23].

\section{Related Work}

A crucial part of understanding what drives embodiment across all ages is understanding the role of movement. Movement provides us with multisensory cues about our own body and surroundings. More specifically, active explorative processes enable us to use multisensory cues to inform us about our body by matching visual, proprioceptive and motor signals of limb movement; and by observing the effects of action on our own body and the environment [24], [25].

These movement-based exploratory processes are already active in three-month-olds kicking a mobile [26], in newborn reaches [27], and in goal-directed foetal movements [28]. We know about the specific sensory correlations which are used and detected in infancy from preferential looking paradigms [29]-[31]. For example, an infant under six months old looks preferentially between a display of legs which move as theirs do, and a display of legs which move out of synchrony (asynchronously) with their own. Thus, the infant detects a visuomotor correlation between their own movement and the asynchronous movement. Likewise, infants detect a visuotactile correlation between viewed and felt touch on a body part [32], [33]. While these sensory building blocks are certainly present in the first year of life, it is not at all certain how they contribute to a subjective sense of self or embodiment [34], [35].

The rubber-hand illusion (RHI) [36] is a popular paradigm used with adults to measure the subjective sense of embodiment (e.g., ownership) for a fake hand. Here, synchronous visuotactile sensory signals (stroking) of the participant's real hidden hand and a viewed rubber hand elicit an illusion where a rubber hand feels like one's own, connected to one's body (i.e., embodiment). The strength of the illusion and corresponding changes in the sense of embodiment, are often seen by increased ratings of ownership, agency and location [2], [36], as well as by 'proprioceptive drift' [37]; a location judgement (given by pointing) where a participant's perceived real hand location shifts from its baseline position towards the rubber hand.

This paradigm has recently been used with children. Results have shown that, like adults, children use visuotactile correlations to drive a sense of hand ownership and location [9], [23], [38], [39]. They also depend more heavily on simpler processes: merely viewing a fake hand near the body elicits a large proprioceptive drift for 4-9 year olds, but not for older children [23], [38], [40]. While informative, the typical RHI paradigm cannot be used to investigate the role of limb movement. For the illusion to occur, participants must keep their hands perfectly still for at least a few minutes, and the illusion is often broken as soon as the participant moves their hand. In fact, many participants spontaneously report that they had "expected the rubber hand to move with them" after the experiment [41]. In light of this, previous research has adapted the RHI with videobased paradigms in order to explore the role of movement, where participant's observe their own hand movements projected live on screen, or delayed [42]-[44].

VR provides an elegant solution to study movement synchrony on embodiment. Using this technology, it is possible to provide participants with a virtual body that moves in or out of temporal synchrony with their own movements. Thus, one can experimentally manipulate sensory information about a moving body in a convincing way. This has been achieved in a range of research over the past decade using optical motion capture or data gloves to track body movement and generate a virtual body that moves synchronously with the user. It has shown that visuomotor synchrony contributes strongly to a sense of hand ownership and location [8], [45]; and can complement visuotactile cues [46] and visual perspective on a body to drive embodiment [47].

VR technologies also offer the opportunity to experience and investigate new virtual selves, where a virtual body may differ in appearance or form to the participant's own. This is crucial for understanding the processes and limitations underlying body representation, showing how incoming visual signals in VR are interpreted against prior knowledge or expectations about how a body should appear. In RHI paradigms, adults do not fully embody a hand with non-human texture [37]. Yet, findings from 'body swapping' illusions suggests that adults embody a virtual body of a different body or gender [48]-[50], skin colour [14], [15], age [51] or size [52]. Research has also found embodiment for a virtual body with modified realism, such as non-corporeal human limbs including a hand with six fingers [53] or an extended arm [54], or non-human forms such as zombie or robot hands [55], [56], or a body with a tail [57].

Since previous research has focused on adults, remarkably little is known about the role of body form in children. Infants have shown sensitivities to the configuration of their own legs [30], while 6-year-olds do not have a sense of ownership for a rubber hand with a different posture to their own [58]. However, in full body VR experiments, five-year-olds embodied a virtual body moving asynchronously to their own [59]. These discrepancies raise important questions for the plasticity of form and body representation in children.

Immersive virtual experiences with a moving body have powerful potential to shape the underlying processes 
of the self, allowing the user to change social attitudes and perceptions [13]-[15], or engage in psychological therapy [50], [60]. There are potential benefits including educational VR experience [61] and pain relief during medical procedures [62]. However, it is important to note the under-researched potential for negative effects, violent or otherwise problematic VR experiences. Research with children has largely focused on locomotor behaviours, affordance judgments and risk [63]-[65], and while useful, little is known about how children perceive and interact within the virtual environment or their virtual bodies [10], [11]. This explanatory 'gap' is problematic given the strong impacts of perceived virtual avatars on social bias and perception known to occur in adults [13]-[15], [66], and the effects of children's social interactions (in VR) with risk behaviours [65]. One example is the Proteus Effect [66], which is the risk that individuals may alter their behaviour to meet the expectations or stereotypes of their virtual avatars.

Further, VR has achieved mass commercial success in recent years, with over 171 million users worldwide, which is set to expand considerably over the coming years [12]. Thus, many children are likely to be exposed to VR despite our lack of an evidence base. The little research available indicates that VR can have a more powerful effect on children than other media. For example, research has shown that children viewing themselves swimming with dolphins in VR could create false memories [67], and that children playing a "Simon Says" game in VR had lower inhibitory control than the equivalent game on a television screen [19]. This very power raises the important ethical issues raised by the use of VR in children, and its impact on their behaviour and development. With a potential for negative effects, it is therefore crucial to understand the impact of VR and virtual embodiment on children; not only for developmental psychologists, but for educators and developers alike.

\section{EXPERIMENT 1}

\subsection{Method}

\subsubsection{Participants}

One hundred and seventeen participants aged 4-14 years $(\mathrm{M}=9$ years, $\mathrm{SD}=2.04)$ were recruited for this study. Of these, there were 34 girls (29\%) and 83 boys $(71 \%)$, and 103 $(88 \%)$ participants were right-handed. All participants reported normal to corrected to normal vision, and reported no sensory, neurological or neurodevelopmental disorders. The study was approved by the local research ethics committee at Durham University (PSYCH-2018-0808T11_21_23-clhv64) and data were collected at the Life Science Centre science museum in Newcastle, UK.

\subsubsection{VR Bubble Popping Task}

A VR funfair environment was developed in Unity (Unity 2018 with VR support enabled for Oculus Rift SDK). All participants took part in a custom-built bubble popping task (see SM A for demo) whilst wearing an Oculus Rift headset (Consumer Version; Menlo Park, CA, USA), and holding an Oculus Touch controller (Oculus, Menlo Park, CA, USA) in their right hand. In the VR environment participants stood in front of a circus tent containing four transparent bubbles in a square formation (Fig. 1), and using their right hand, had to place a virtual hand through the bubble that lit up to a vibrant blue colour. The bubbles turned blue in a random order every 2 seconds, with only one bubble lit at any one time. This elicited a controlled movement at a consistent frequency, ensuring that movement experience was consistent across participants and conditions.

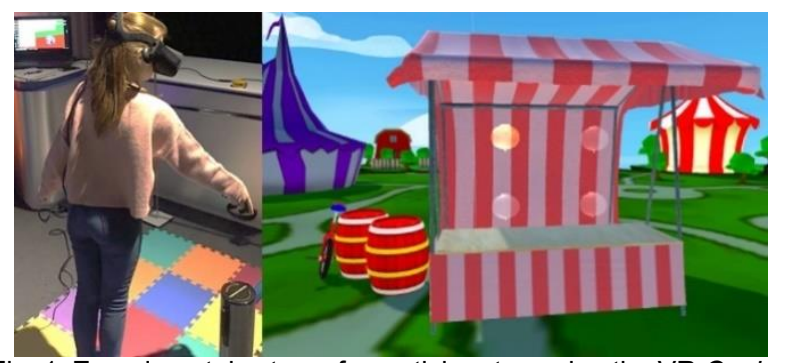

Fig. 1. Experimental set up of a participant wearing the VR Oculus Rift headset (left) and the funfair bubble popping environment (right).

Participants completed a 60-second 'practice' trial of synchronous bubble popping where their virtual hand movements were screen-recorded. This gave participants practice with the task and provided a recording of their movement which was used as a playback video. There were two movement trials where the participant's real and virtual hands were either temporally synchronous or asynchronous (see SM B for schematic). During synchronous movement, the virtual hand moved in real time with the participant's own movements. During asynchronous movement, the virtual hand was driven as per the pre-recorded movements from the practice trial. This meant that during asynchronous trials, while participants saw a new order of bubbles lighting up, they were unable to control the virtual hand. It did not respond to the participant's own hand movements and the observed and felt movement were temporally and spatially incongruent. We also explored the effects of haptics on embodiment of the virtual hand. Participants received haptic feedback (a 'buzz') from the controller in their right hand for a duration of 1 second. Participants were allocated to one of three haptics conditions: congruent haptics (haptic sensation was matched to when the user popped a bubble), delayed haptics (a delayed buzz was felt randomly between 1-2 seconds after popping a bubble) or no haptics (no buzz felt).

For the hand model, we used the VR hands and FP Arms Pack by NatureManufacture ${ }^{1}$. These were realistic representations of a human child's hand (female), and this same model was used for all participants (Fig. 2). It was scaled to each participant's hand size, which was measured (in $\mathrm{cm}$ ) on the right hand, palm upwards, from the tip of the middle finger to the first crease of the wrist. The hand had a simplified skin texture, corresponding to 
relatively light skin which matched the majority of our participants. Participants' hands were tracked in 6 degrees of freedom (position and rotation); in the synchronous condition this was mapped directly onto the hand model's position and rotation. There was no other form of hand tracking, and the fingers on the hand did not move. Participants were asked to grip the controller for the entire study and the finger posture of the hand model corresponded to a grip, so the virtual and real fingers approximately corresponded in posture. At all times, the virtual hand was offset from the real hand by $25 \%$ percent of each participant's arm length (see section 3.1.4).
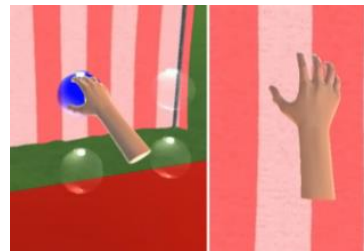

Fig. 2. Demonstration of the virtual hand (set here to size $12 \mathrm{~cm}$ ). From left: hitting a bubble, palm facing down, and palm facing left at approx. $90^{\circ}$ and $45^{\circ}$ tilted up.

\subsubsection{Questions}

At the end of each bubble popping trial, participants were asked to rate their level of ownership and agency for the virtual hand. A control question was included to check whether participants were answering appropriately (see Table 1). These questions were based on previous similar measures of embodiment [2], [41] and our own work with children [9], [23], [38], [58]. For all questions, participants used a virtual laser beam whose origin was at the virtual hand to select a rating from a continuous response scale, ranging from 0 ("no, definitely not") to 1 ("yes, lots and lots"). Questions were simultaneously read aloud twice to the participants to ensure understanding [23]. Participants were reminded before answering the questions that they could respond anywhere along the scale.

Table 1. Embodiment questions for Exp. 1

Questions for Exp. 1

Q1 Did you sometimes feel like the virtual hand was your hand or belonged to you?

Q2 Did you sometimes feel like you were moving the virtual hand?

Q3 Did you sometimes feel like your leg turned green?

\subsubsection{Proprioceptive Drift}

Measures of proprioceptive drift are dominant in RHI studies [23], [37], [38], [58] and were included here to assess how VR experience affected the participant's perceived hand location. The virtual hand was offset by $25 \%$ of the participant's arm length (this was measured in $\mathrm{cm}$ from the top of the shoulder to the tip of the middle finger of the right hand). The offset direction was set along the $x-$ axis (running along the participant's left-right axis), towards the centre line of the body. This body-relative scaling was done to ensure that motor demands were the same for differently sized participants and replicates previous tabletop RHI studies [23], [38], [58]. A 1.2m wide horizontal pole clamped between two vertical poles (" $\mathrm{H}$ " shape with a long centre line) was placed in front of participants and height-adjusted up or down to be level with each participant's navel. A left Oculus Touch controller was attached to the horizontal pole, so that, guided by the participant's left hand, it could easily slide from left to right to act as a pointer. Following movement trials, the HMD displayed a black screen and participants, with their eyes closed, were asked to indicate (using the slider, with their left hand) the perceived position of their right hand. This measure was taken twice at baseline (averaged in analysis) before any VR exposure, and once after each movement condition. For each participant, the baseline pointing position was subtracted from the post-movement pointing position, and the result divided by the participant's arm length, to give an estimate of proprioceptive drift as a percentage of arm length [23].

\subsubsection{Procedure}

After obtaining verbal assent from the child and written consent from their carer, participants were randomly assigned to a haptic condition: congruent $(n=40)$, delayed ( $n$ $=37)$ or none $(n=40)$, with age range kept similar across conditions. To begin, participants completed two baseline measures for proprioceptive drift outside of VR. The participant entered the virtual environment while holding both Oculus Touch controllers, while the pole structure was moved out of range during the bubble popping task. Next, they completed a brief training session of the rating scale (using the sliding virtual response scale to rate familiar foods). They then completed one block of three movement trials, each lasting 60 seconds. This included: one practice trial, one synchronous movement trial and one asynchronous movement trial, while receiving the haptic feedback assigned to them. The practice trial was always completed first, while the order of movement trials was counterbalanced across participants. After each movement trial, participants were asked to make the location judgement using the pole structure (this was used to calculate proprioceptive drift), and then answer questions (Table 1) in VR pertaining to the movement trial they had just experienced. The entire experiment took approximately 20 minutes. Both carer and child were debriefed afterwards.

\subsection{Results}

All analyses were conducted on a full sample of 117 participants $(M=9$ years, $S D=2.04)$. The average response to the control question was calculated for each participant, which revealed that 13 participants $(11 \%)$ answered at the halfway point (0.5) or above. However, we noted during the experiment that participants might be confused about the inclusion of the colour 'green' in the question, since the virtual ground (and environment) was also green. As we could not be certain which participants were responding appropriately to this question, we did not use it to calibrate responses given to the other questions [9].

We found that children were not using the full range of the scale, but rather clustering their responses around the extremes ( 1 and 0$)$. Before modelling the data, we therefore transformed responses into low (lower than 0.5) and high (greater than or equal to 0.5) groups. We used a binomial logistic regression in a generalized linear mixed model (GLMM), implemented via the lme4 package [68] in $R$ 
(version 3.6.0, [69]). GLMMs were fit to each of the questionnaire items (ownership and agency). Each model included effects of movement (synchrony vs asynchrony), haptics (no haptics, congruent and delayed), and movement by haptic interactions, controlling for age. All figures were produced in R package ggplot2 [69], [70] and present medians and interquartile ranges (IQR) overlaid with individual data points. GLMM output tables can be found in SM C. Post-hoc analyses were computed using non-parametric tests (Kruskal-Wallis and Spearman's rank test), and any multiple comparisons were controlled for using the False Discovery Rate [71].

\subsubsection{Ownership}

Ownership ratings are presented in Fig. 3 (SM C1). In models with only movement or haptics as a predictor alongside age there was no effect of either and no interactions of either with age (odds ratios [ORs] $=0.63-1.86, p^{\prime} \mathrm{s}>.065$ ). When entering both movement and haptics together, the main effect of movement became significant $(\mathrm{OR}=0.38, p$ $=.043)$, with higher ratings in the synchronous condition. However, given this result was non-significant in all other models, we do not consider it robust. There were no other significant effects or interactions (ORs 0.99-2.35, p's >.064).

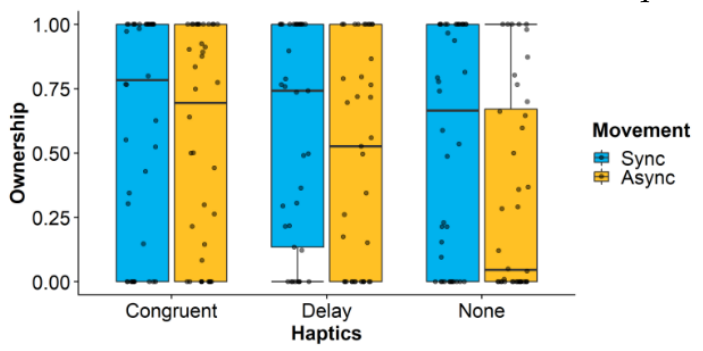

Fig. 3. Ownership ratings (medians and IQRs) overlaid with individual data points for both movement conditions in all haptic conditions.

\subsubsection{Agency}

Agency ratings are presented in Fig. 4 (SM C2). In models with only movement or haptics as a predictor, there was a main effect of movement $(\mathrm{OR}=0.13, p<.001)$, with higher ratings in the synchronous condition (in all haptic conditions, all Z's $\left.>-2.74, p^{\prime} \mathrm{s}<.006\right)$. There was a main effect of haptics on scores in delayed $(\mathrm{Mdn}=0.96, \mathrm{OR}=2.33, p<$ .029 ) but not congruent haptic conditions ( $\mathrm{Mdn}=0.89$, OR $=1.62, p=.172)$. Age interacted with movement $(\mathrm{OR}=0.58$, $p=.016)$ but not haptic conditions (ORs 0.67-0.90, $p^{\prime} \mathrm{s}>$ .078). When entering both movement and haptics together, these effects remained, except the effect of delayed haptics became non-significant $(\mathrm{OR}=2.76, p=.058)$. Given this result was present in all other models, it was explored via post-hoc analyses (Kruskal-Wallis test), which revealed no significant differences in haptic conditions $\left(\mathrm{H}^{\prime} \mathrm{s}<3.73, p^{\prime} \mathrm{s}\right.$ $>$.155). In this model there were no movement by haptics interactions (ORs 1.24-1.67, $p^{\prime}$ s > .554).

Post-hoc analysis of the age by movement interaction using Spearman's rank correlation revealed a negative relationship between age and agency scores in the asynchronous condition only, $r_{s}(117)=-.26, p<.01$ (synchronous condition, $p=.117$, see SM D1). However, since the $\mathrm{R}^{2}$ value was low (0.06), age cannot reliably explain a large portion of variability in the scores. portion of variability in the scores. May seem surprising, similar findings have been observed
1077-2626 (c)

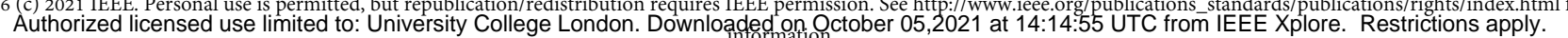

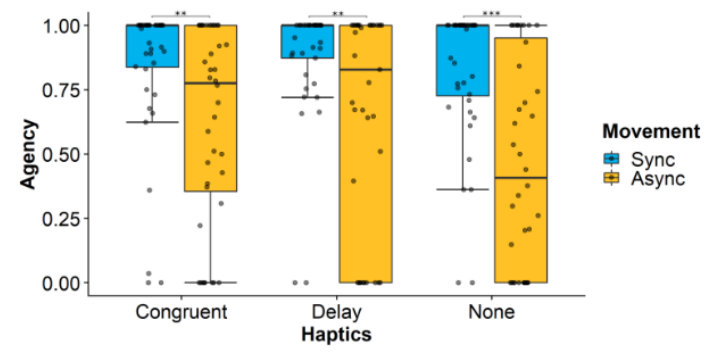

Fig. 4. Agency ratings (medians and IQRs) with overlaid individual data points for synchronous and asynchronous movement in the three haptic conditions: congruent, delayed and none $\left({ }^{* *} \mathrm{p}<.01\right.$, ${ }^{* * *} \mathrm{p}<$ $.001)$.

\subsubsection{Proprioceptive Drift}

For proprioceptive drift, measures were analysed using a 2 (movement: synchronous, asynchronous) x 3 (haptics: conage (see SM E for data). The age covariate was mean-cenCOVA [72]. There were no effects or interactions of any factor on drift $\left(F \mathrm{~s}<1.57, p^{\prime} \mathrm{s}>.213, n_{p}^{2 \prime} \mathrm{s}<.027\right)$.

\subsection{Discussion}

Findings from Exp. 1 indicate that participants felt sensations of embodiment for a virtual hand which was predicted by movement synchrony, supporting previous research that congruent movement (i.e., visuomotor signals) facilitates embodiment [8], [45]. Here, however, an effect of movement synchrony was only observed for agency, and not ownership. This result was unexpected, and we note that ownership was, overall, rated substantially lower than agency. The fact that the ownership question was comprised of two parts using an "or" statement may have caused some confusion.

Agency and ownership ratings were comparable in all facilitate or effect embodiment. This was unexpected and in contrast to previous findings that congruent haptics (touch) elicit increased embodiment for virtual hands [7], [36], [48], [73]. One possible reason for this is that particiOculus Touch controllers, and not on their own body [23], [36], [48], [73], [74]. Secondly, participants did not observe any simultaneous visual input of the tactile sensation as in previous experiments (e.g., the sight of a brush [36] or a tapping ball [74]), which likely signifies and facilitates attentional processes to the localization of the touch. Thirdly, the action of the task (bubble popping) may not have matched the expected tactile sensation; the controller was held with a clenched fist, yet haptic feedback was localised to the centre of the controller. Instead, one might expect tactile sensation in the finger or the part of the hand that first contacted the bubble. Thus, the present task, along with current haptic hardware (Oculus Touch) may not be sufficient to generate this type of illusion.

The negative relationship between age and asynchronous agency suggests that on average, younger children were unaware or less affected by incongruent visuomotor signals compared to older children. Although this result gruent, delayed or none) mixed ANCOVA, controlling for tered, based on guidelines for repeated measures ANhaptic conditions, suggesting that tactile sensation did not pants received tactile stimulation indirectly through the 
in children [59] and certain adult populations [41], [75]. However, given the low $\mathrm{R}^{2}$ value, we note age is unlikely to explain a large portion of variability in agency scores.

Finally, while we expected to see a shift in proprioceptive drift under conditions of movement synchrony as per previous findings [23], [37], [38], there were no differences in drift for any condition. One possibility is that, unlike previous research with children [23], [38], [58], proprioceptive drift was calculated by the distance between the two Oculus Touch controllers, held with clenched fists. The relatively large controllers may have resulted in less precise measurements and the hand clenching may have given proprioceptive signals to current hand position. Further, the posture of the real and virtual hands may have been slightly mismatched, which can cause less drift in the tabletop RHI for children as well as adults [58].

\section{EXPERIMENT 2}

For this experiment we explored the effects of the appearance (i.e., form) of the virtual body on sensations of embodiment in children. Previous research has found that sensations of embodiment are stronger in adults who view a rubber or virtual hand with a similar appearance to their own (human) hand [37], [76], [77]. Thus, sensations of embodiment are generated when incoming visual information is congruent with prior knowledge or reference [78]. However, this has been challenged given that individuals can embody various virtual bodies or limbs dissimilar to their own. For example, participants can embody a virtual body that differs to their own in terms of appearance [48], age [51], or one that is positioned physically incongruent to their own [79]. Further, embodiment has been observed for virtual objects that do not portray a typical or human functional representation. A skin-like texture has been found to be helpful, but not necessary for embodiment [80], yet embodiment has been observed (to varying degrees) for non-corporeal or non-human forms, such as a hand with six fingers [53] and a body with a tail [57]. However, such factors are yet to be explored with children - particularly for moving virtual bodies.

In the current study we used a modified version of the VR bubble popping task in Exp. 1 where participants used either a virtual hand or a virtual 3D block (cross) to place their hand through the bubbles (see Figs. 2, 5). This manipulated the effects of human form (virtual appearance) of the virtual hand and investigated whether participants would feel a sense of embodiment for a virtual limb of a different shape, with different functional connotations to a human hand.

In addition, we included and adapted several new questions pertaining to overall embodiment (see Table 2) to gain a better understanding of the precise sensations felt while moving the virtual body. These were adapted from previous research on embodiment [2], [41] and children [9], [23], [38], [58]. The response scale was modified to a "scores out of $10^{\prime \prime}$ scale to try and achieve a greater range of responses, and to ensure that they were more relatable for young children, and more easily interpreted by the experimenters. For example, a 5 out of 10 being sufficiently less than a score of 10 out of 10 . Each child took part in a brief verbal question training session where they were asked to rate items out of 10 (e.g., homework and different foods). The control question from Exp. 1 was adapted since we could not ascertain whether participants had been influenced or confused by the green ground (and environment) within the HMD.

\subsection{Method}

\subsubsection{Participants}

Eighty participants aged 5-14 years $(M=9$ years, $S D=$ 2.00) were recruited for the study. Of these, there were 33 girls (41\%) and 47 boys (59\%), and $66(83 \%)$ reported being right-handed. All participants reported having normal to corrected to normal vision, and no sensory, neurological or neurodevelopmental disorders. The study was approved by the local research ethics committee at Durham University (PSYCH-2018-08-08T11_21_23-clhv64) and data were collected at the Life Science Centre, Newcastle, UK.

\subsubsection{VR Bubble Popping Task}

Each participant completed the VR bubble popping task described in Exp. 1, with some modifications. Half of the participants used a virtual hand as described in Exp. 1, and the other half used a virtual "block", a 3D cross (Fig. 5). The virtual block was adapted from the virtual hand pack as described in Exp. 1. Like the virtual hand, it was scaled to each participant's hand size and had a simplified skin-like texture. This represented a similar skin colour to most of our sample, but on a non-human form / structure. As in Exp.1, participants' hands were tracked in 6 degrees of freedom (position and rotation) and mapped onto the hand model's position, but the cross was rotated by 45 degrees (Fig 5). Participants experienced both synchronous and asynchronous movement conditions, but here they completed two trials for each movement type. Lastly, there was no haptic feedback at any time during the experiment.

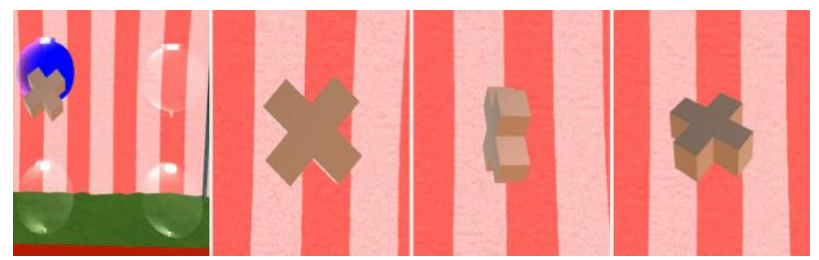

Fig. 5. Demonstration of the virtual block (set here to size $15 \mathrm{~cm}$ ). From left: hitting a bubble, cross (palm) facing down, rotated to the left approx. $90^{\circ}$ and tipped upward slightly.

\subsubsection{Questions}

Participants were asked to respond to five questions considered here as general 'embodiment' ratings and a new control question (Table 2). For all questions, participants answered on a rating scale from 0 ("not at all") to 10 ("definitely yes"). We included two questions on ownership; a question on agency; a question pertaining to location; and a question that asked participants to think specifically about their feelings of ownership over the virtual form; choosing whether it felt more like a hand or more like a tool (mouse / controller). Note, responses to Q5 were reverse-scored: high scores indicate that the virtual hand or block felt like their own hand (Fig. 8). 


\section{Table 2. Embodiment questions for Exp 2}

\begin{tabular}{lll} 
& Questions for Exp. 2 & Component \\
\hline Q1 & The hand / block felt like my own hand & Ownership \\
Q2 & The hand / block felt like part of my own body & Ownership \\
Q3 & I was in control of moving the hand/ block & Agency \\
Q4 & $\begin{array}{l}\text { My own real hand was in the same place as the hand / } \\
\text { block }\end{array}$ & Location \\
Q5 & $\begin{array}{l}\text { The hand / block felt more like a mouse or controller than } \\
\text { part of my own body }\end{array}$ & Tool-use \\
Q6 I felt like my leg turned purple & Control
\end{tabular}

\subsubsection{Proprioceptive Drift}

Measures of proprioceptive drift were as described in Exp. 1 , except here, instead of using a pole structure, participants were seated throughout the experiment in front of a small table holding both controllers, and asked to slide their left controller under the table to the location of their right hand, mirroring previous tabletop studies with children [23]. This ensured that drift measures were taken as soon as the bubble popping trial had finished and allowed more precise and controlled localisation judgments underneath the table. Drift measures were taken after each movement condition.

\subsubsection{Procedure}

This was the same as Exp. 1 with the following exceptions. Firstly, there were no haptic conditions. Secondly, each participant was randomly assigned to either the hand $(n=$ $40)$ or block form $(n=40)$ group, with age range kept similar across conditions. Thirdly, participants completed two trials of each movement type rather than one. Thus, participants completed one block of five trials, each lasting 60 seconds: including one practice trial, two synchronous movement trials and two asynchronous movement trials. The practice trial was always completed first. Next, half the participants completed the two synchronous trials followed by the two asynchronous trials, while the other half completed the two asynchronous trials first. Each movement trial was followed by a pointing estimate, and these two points for each movement type were averaged in analysis of proprioceptive drift. Participants answered one set of questions (Table 2) after completing two synchronous movement trials and one set after the two asynchronous trials.

\subsection{Results}

Participants' responses to the control question after each movement condition were averaged in analysis, and participants scoring above 5 out of 10 were removed from analysis. This resulted in five participants $(6 \%)$ being removed and a final sample of 75 participants with 32 $(43 \%)$ girls, range $5-14$ years, $M=9$ years, $S D=2.04,64$ $(85 \%)$ right-handed.

Ratings were more evenly spread than in Exp. 1 but were still non-normal. We therefore present medians and IQRs in all figures, overlaid with individual data points. The data were transformed using Aligned Rank Transformation (ART [81]) prior to parametric analysis. This procedure first aligns and ranks the raw data which can then be submitted to standard parametric analysis. It allows for an examination of both main and interaction effects without the contamination of other variables and has been used in similar embodiment studies using rating scales [9], [58]. The transformed data were then analysed using a 2 (movement: synchronous, asynchronous) $\times 2$ (form: hand, block) mixed measures ANCOVA, with age as a mean-centred covariate [72]. Post-hoc comparisons were analysed via non-parametric tests on the untransformed data (Wilcoxon signed-rank and MannWhitney $U$ tests).

\subsubsection{Ownership}

For ownership Q1 (Fig. 6a) there were main effects of movement $F(1,72)=24.34, p<.001, n_{p}^{2}=.253$, and form $F(1,72)=4.75, p<.05, n_{p}^{2}=.062$, with higher ratings for synchronous movement and the hand form. The movement by form interaction was significant $F(1,72)=5.59, p$ $<.05, n_{p}^{2}=.072$. Other effects and interactions were not significant $\left(F \mathrm{~s}<1.49, p^{\prime} \mathrm{s}>.227, n_{p}^{2}\right.$ s $\left.<.031\right)$. Exploring the form by movement interaction, Wilcoxon signed-rank tests revealed higher ratings for synchronous $(\mathrm{Mdn}=5.78)$ than asynchronous (Mdn $=1.50)$ movement for the block $Z=$ 3.58, $p<.001, r=.29$, but not the hand $Z=-1.79, p=.073, r$ $=.15$. Mann-Whitney $U$ tests revealed no difference between forms in synchronous $U=524.00, p=.06, r=.22$, or asynchronous $U=690.00, p=.884, r=.02$ movement conditions.

For the second ownership question $(\mathrm{Q} 2$ : Fig. $6 \mathrm{~b})$ there was a main effect of movement only $F(1,72)=38.12, p<$ $.001, n_{p}^{2}=.346$, and no other significant effects $(F \mathrm{~s}<2.10$, $\left.p^{\prime} \mathrm{s}>.152, n_{p}^{2}<.028\right)$.
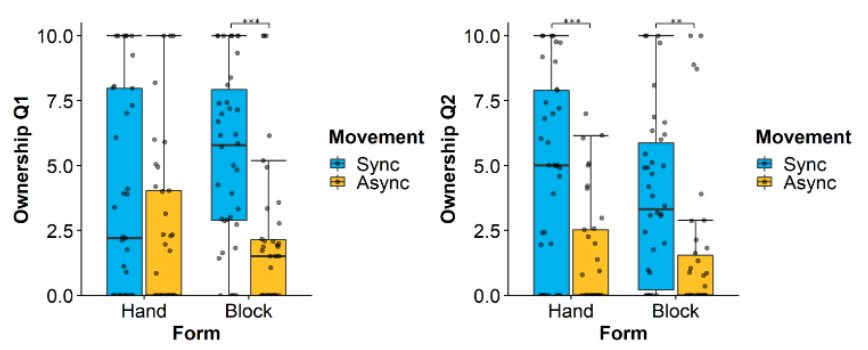

Fig. 6. Ratings (medians and IQRs) for (a) Ownership (Q1) and (b) Ownership (Q2) with individual data points for the hand and block forms during movement conditions $\left.{ }^{* *} p<.01,{ }^{* * *} p<.001\right)$.

\subsubsection{Agency}

For agency Q3 (Fig. 7a) there was a main effect of movement, $F(1,72)=124.18, p<.001, n_{p}^{2}=.633$, with higher ratings in the synchronous condition. There were no other significant effects $\left(F \mathrm{~s}<2.64, p^{\prime} \mathrm{s}>.109, n_{p}^{2}<.039\right)$.

\subsubsection{Location}

For location Q4 (Fig. 7b) there was an effect of movement, $F(1,72)=79.21, p<.001, n_{p}^{2}=.524$, with higher ratings in the synchronous condition, and an effect of the covariate age, $F(1,72)=5.99, p=.017, n_{p}^{2}=.077$. No other effects were significant $\left(F \mathrm{~s}<2.98, p^{\prime} \mathrm{s}>.06, n_{p}^{2}\right.$ 's $\left.<.076\right)$. Spearman's correlation revealed that age negatively correlated with synchronous movement, $r_{s}(75)=-.31, p<.01$, but not asynchronous movement $(p=.126)$. However, as the $\mathrm{R}^{2}$ value was low (0.05), age cannot explain a large portion of the variability in location scores (see SM D2). 

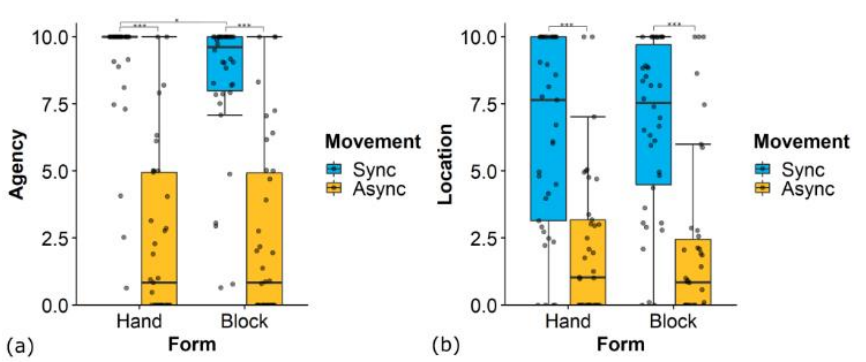

Fig.7. (a) Agency (Q3) and (b) Location (Q4) ratings (medians and IQRs) with individual data points for the hand and block forms during synchronous and asynchronous movement $\left({ }^{*} p<.05\right.$, $\left.{ }^{* * *} p<.001\right)$.

\subsubsection{Tool-Use}

For tool-use Q5 (Fig. 8) there were effects of movement, $F(1,72)=9.31, p<.01, n_{p}^{2}=.115$, and form $F(1,72)=5.62, p$ $<.05, n_{p}^{2}=.072$. Ratings were higher in the synchronous movement condition and for the hand form. The movement by form interaction was also significant $F(1,72)=$ $5.45, p<.05, n_{p}^{2}=.070$. No other effects were significant $(F \mathrm{~s}$ $<2.73, p^{\prime} \mathrm{s}>.103, n_{p}^{2}$ s $\left.<.037\right)$.

Exploring the interaction between movement and form, Wilcoxon signed-rank tests revealed a significant effect of movement synchrony for the hand, $Z=-3.07, p<.01, r=$ .25 , but not for the block, $p=.837$. Compared to the asynchronous movement condition, the synchronous movement condition elicited higher ratings, but only when the virtual form was a hand. Mann-Whitney $U$ tests revealed higher ratings for the hand compared to the block in synchronous movement, $U=416.00, p<.01, r=.35$, but not in asynchronous movement, $p=.741$. Compared to the block, the virtual hand was rated more like a body part and less like a tool, but only with synchronous movement.

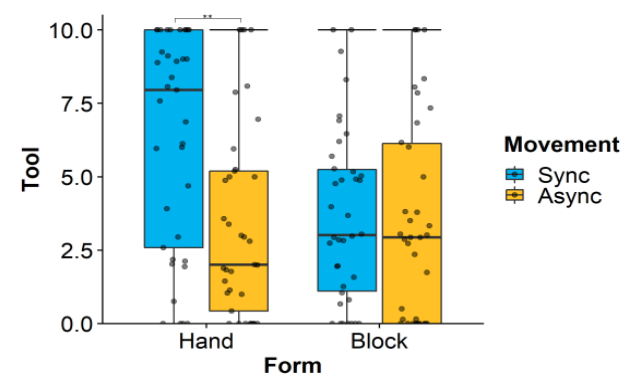

Fig. 8. Tool-use (Q5) ratings (medians and IQRs) with individual data points for the forms during both movement conditions. Higher scores indicate feeling that the virtual form was more like a body part; lower scores that it was more like a mouse or controller $\left({ }^{* *} p<.01\right)$.

\subsubsection{Proprioceptive Drift}

Proprioceptive drift measures analysed via an ANCOVA revealed no main effects of movement or form, nor any interaction between movement or form $\left(F \mathrm{~s}<1.47, p^{\prime} \mathrm{s}>\right.$ $.230, n_{p}^{2 \prime}$ s $\left.<.020\right)$. There were no effects or interactions with the covariate age $\left(F \mathrm{~s}<2.72, p^{\prime} \mathrm{s}>.103, n_{p}^{2 \prime} \mathrm{s}<.036\right.$, see SM E).

\subsection{Discussion}

For all questions (ownership, agency, location, and tooluse) there was a significant effect of movement, where synchronous movement generated higher ratings of embodiment compared to asynchronous movement. This supports previous findings of congruent visuomotor synchrony driving virtual embodiment [8], [45]. Interestingly, there was an effect of movement synchrony for a virtual block as well as a virtual hand, supporting previous findings of embodiment for non-human forms [17], [55]-[57], [82]. The hand and block were rated similarly in terms of ownership, agency, and location. The findings from the tool-use question revealed a difference between forms. Movement synchrony caused the virtual form to be rated more like a body part than a tool, but only for the hand, not the block. Likewise, the hand was rated as more like a body part than the block during synchronous movement only. Therefore, the combination of synchronous movement and a hand-like form was special in driving a feeling that the virtual form was not just a mouse or controller, but part of the participant's own body.

For most questions, there were no effects of age. However, there was a negative relationship between age and location ratings in synchronous movement, suggesting that younger children felt more like their hand was in the same location as the virtual one compared to older children. This supports previous findings that movement synchrony facilitates a change in perceived hand location [8], [23], [36], except here, a difference between young and older children was observed. However, as per Exp. 1, the low $R^{2}$ value suggests that age cannot explain a large portion of the variability in scores. Further, location judgements made by pointing (proprioceptive drift measures) did not correlate with age, which suggests that here, perceived subjective location and perceived drift were dissociable (as has been suggested by [83]).

Finally, despite adapting our measure of proprioceptive drift (i.e., participants were seated at a table and moved their hands along the table) to allow a more controlled and precise movement, there were no differences in drift for any condition. We therefore reiterate our points from Exp. 1; that drift calculated between the two controllers, while holding them with a clenched fist, is insufficient and less precise compared to standard RHI paradigms and previous experiments with children [23], [38], [40], and likely due to the fact that holding the controllers provides significant proprioceptive information about the current position of the arm.

\section{General Discussion}

These experiments reveal that children from 4 years old felt a sense of embodiment for a virtual agent when it moved synchronously (i.e., congruently) with their own movements. This was observed in two independent samples of children aged between 4-14 years via ratings pertaining to a sense of ownership, agency, and hand location. These findings of movement synchrony support previous work with the static RHI paradigm with children [9], [23], [38], [39] showing that multisensory signals drive children's embodiment, but crucially extend this to visuomotor synchrony. The findings suggest that, like adults [8], congruent visuomotor signals strongly drive children's embodiment [8], [45]. 
Both experiments also revealed that users gave the highest ratings for a sense of agency, with the effects of movement on agency also being very pronounced. While agency and ownership are often considered interrelated subcomponents of embodiment, our findings underline previous research suggesting that they represent distinct cognitive processes [84].

We note that ratings of ownership, agency, and location were comparable for the hand and block forms. Likewise, the effect of visuomotor synchrony was present even for the block (Exp. 2). These findings would seem to support previous research with adults showing embodiment for non-corporeal [17], [53], [82] or non-human forms [55]-[57] and extend them to children. Importantly though, there was also a clear difference observed between forms when children were asked to think about whether the virtual form felt more like a body part or a mouse/controller. When attention was drawn to this distinction, only the synchronously moving hand was rated like a body part rather than a tool. This suggests that ownership was driven by form only subtly. The obvious contrast with the consistent effects of movement lends support to more bottom-up accounts of body representation which emphasize congruent multisensory signals, rather than prior knowledge, as primary drivers of embodiment [7], [37].

In Exp. 1 we found a selective, negative relationship between age and agency ratings in the asynchronous movement condition. We note that the $\mathrm{R}^{2}$ value was low, suggesting that while age was, on average, associated with higher scores, it does not explain a large portion of variability. However, we also note that similar findings have been observed in children during a full body illusion [59], where 5 year-olds report embodiment for a virtual body under asynchronous movement. These results support the notion that the default is to accept a body seen from a firstperson perspective as one's own until evidence is provided to the contrary [47], [59], [83]. This tendency may be most pronounced in the youngest children, implying more plastic body representation at this age. We note that there were more younger children in Exp 1 (17 children $\leq 6$ years) than in Exp 2 (7 children $\leq 6$ years, see SM D3). Further work should examine the age dependence of this perspectivedriven body acceptance in very young children.

Finally, we found no differences in proprioceptive drift measures in either experiment. This goes against previous research on similar illusions with adults, e.g. in the RHI [36], [37] and previous work with children [23], [38]. One reason for this might be that previous studies measured drift in a precise tabletop version where the participant's hand position is tightly controlled. Despite changing our measurement of drift from a standing (Exp. 1) to seated position (Exp. 2) there were no differences in drift for any condition. Our measures of drift were taken via two points on the Oculus Touch controllers, which may still lack the necessary precision in such a task. Further, in the RHI paradigm the hands are usually placed flat (unclenched) and left to rest for several minutes before a drift measurement is taken. In the current experiments, participants were actively gripping the controllers at the time the drift measure was taken and were likely receiving at least some feedback of their hand location. Finally, the postures of the controller and virtual hand may be difficult to match exactly. We therefore conclude that drift cannot be used to measure embodiment in the current setup.

\subsection{User Implications}

There are several notable implications from this research within the context of VR applications, such as in education or gaming. Firstly, in both experiments we observed that children's body representation can be temporarily manipulated within a virtual environment if the movement between the virtual hand / body and participant's own body are visually, spatially, and temporally matched. Secondly, all children (aged 4-14 years) felt some sense of embodiment for a virtual object with properties that looked both similar (a human hand) and strikingly dissimilar (a nonhuman block) to their own hand. This was true for ratings of ownership, agency, and location. Thirdly, children did feel that a non-human form was more like a tool than a body part. Finally, the findings highlight the importance of visuomotor synchrony over haptics for feelings of embodiment, when applied using current commercially available hardware. Haptic feedback, however, may well be important for presence or embodiment in other VR tasks where the felt touch is at a precise location in relation to the action or manipulation of an object (e.g., haptic feedback on the tip of the index finger to pop a bubble), or where more typical touch sensations are used [46].

More broadly, this research indicates some beneficial effects of VR embodiment that could transfer to children's education. For example, research has found that embodying an avatar of a different skin tone can reduce implicit racial bias [14], [15]. Our findings, however, also highlight the risk of potentially negative VR embodiment effects (e.g., the Proteus effect [66]), and raise questions on the responsibility and demand for increased awareness in VR usage. If young children can feel ownership and a sense of agency (i.e., embodiment) for a neutral virtual block, what are the implications for feeling these sensations for something virtually violent, such as weapons or performing violent acts? Another area of concern is body image. If the results presented in this paper extend to full body ownership, what are the potential risks on children's body image if embodying unrealistically thin or in other ways stereotypically attractive avatars? In both cases, the effects of VR may well be much stronger than non-immersive, non-embodied media representations.

\subsection{Limitations}

The current experiments have some limitations which we will discuss in turn. Firstly, in relation to participant recruitment. We note there was an unequal balance of gender in Exp. 1 with only $29 \%$ girls. Gender differences have been found in VR experiences for simulator sickness or variability in controller grip (for review see [85]). Thus, the interpretation and generalization of findings should be considered accordingly. However, we note the main findings of movement synchrony were observed within-subjects, and thus not affected by gender. In addition, the gender balance was improved in Exp. 2, with 41\% girls recruited, with key findings of movement synchrony replicated here. There 
was also a greater prevalence of right-handers in both Exp. $1(77 \%)$ and Exp. 2 (83\%). However, we chose to have all participants use their right hand with the controller because assessing effects of handedness would be difficult in a sample of this size. Further, left-handed children might use either hand when performing daily tasks like ours (e.g., throwing a ball).

Secondly, to enable us to measure drift, the virtual hand was offset for each participant. However, greater distance between a real and fake hand has been associated with attenuated ownership [86]. Since virtual hands were uniformly offset in all conditions, this is unlikely to have affected the findings observed between movement conditions or the different virtual forms. However, it is likely that the embodiment of virtual hands would be higher overall in a virtual context where the real and virtual hands are spatially aligned.

Thirdly, we note embodiment was quantified via only two questions in Exp. 1 and five questions for Exp. 2. While we would ideally explore the components of embodiment more thoroughly, this is not suitable for children. In designing our questions, we selected a small number to keep the experiment engaging for small children and to avoid dropout. Likewise, we ensured that the questions used short, simple phrasing, which piloting has shown to be understood by the youngest participants in our sample. Questions were presented in written form but simultaneously read aloud to each participant [23]. In addition, our formulations very closely match those that are prevalent in adult studies [2], [36], [41], [87], [88], and our own work with children [9], [23], [38], [58]. Together these design features make us satisfied that our question choices were largely reasonable, comprehensible, and useful.

One final limitation concerns the control questions. While our Exp. 1 question was based on a similar hair colour question [59], asking participants if their legs were green in this particular grassy scene led to misunderstanding. Further, as there were no legs visible in the virtual scene, this may have added confusion, resulting in the removal of $6 \%$ of respondents even in Exp. 2. This issue highlights the need for standardized questions when exploring VR embodiment, for children as well as adults: we note that standard adult questions are increasingly available in the literature [87], [88]. However, given that the remaining questions showed meaningful response patterns in two independent samples, we are confident that children were providing nuanced responses rather than answering at random or agreeing across the board.

\section{Conclusion}

Collectively, our findings highlight the importance of movement synchrony for children's feelings of embodiment - shown here for moving virtual hands. Embodiment (measured via ratings of ownership, agency, and location) was observed for both a virtual hand and nonhuman form (block) to some degree, providing visuomotor signals were congruent. This ownership for a non-human virtual object highlights the dominant role of congruent visuomotor and proprioceptive signals in own-body representation. However, only the hand in the synchronous condition was described as feeling like part of the body rather than a tool, demonstrating that embodiment is also somewhat constrained by prior expectations of body form. The work signals the strong potential of embodied VR with children.

\section{ACKNOWLEDGMENT}

This work was funded by the Economic and Social Research Council (ESRC, ES/P008798/1 to Dr Cowie) and the Swedish Research Council (VR-PG 2017-01504 to Dr Gottwald). The authors wish to thank Prof. Lynda Boothroyd for guidance on statistical analyses; the children who volunteered for these experiments; and the Life Science Centre in Newcastle, UK for hosting us. *Dr Dewe \& Dr Gottwald contributed equally to the work and correspondence should be addressed to either.

\section{REFERENCES}

[1] S. Gallagher, "Philosophical conceptions of the self: Implications for cognitive science," Trends Cogn. Sci., vol. 4, no. 1, pp. 14-21, 2000.

[2] M. R. Longo, F. Schüür, M. P. M. Kammers, M. Tsakiris, and P. Haggard, "What is embodimnent? A psychometric approach," Cognition, vol. 107, pp. 978-998, 2008.

[3] O. Blanke and T. Metzinger, "Full-body illusions and minimal phenomenal selfhood," Trends Cogn. Sci., pp. 7-13, 2009.

[4] K. Kilteni, R. Groten, and M. Slater, "The Sense of Embodiment in Virtual Reality," Presence Teleoperators Virtual Environ., vol. 21, no. 4, pp. 373-387, 2012.

[5] K. Suzuki, S. N. Garfinkel, H. D. Critchley, and A. K. Seth, "Multisensory integration across exteroceptive and interoceptive domains modulates self-experience in the rubber-hand illusion," Neuropsychologia, vol. 51, no. 13, pp. 2909-2917, 2013.

[6] M. Tsakiris, "My body in the brain: A neurocognitive model of body-ownership," Neuropsychologia, vol. 48, no. 3, pp. 703-712, 2010.

[7] H. H. Ehrsson, "43. The Concept of Body Ownership and Its Relation to Multisensory Integration," New Handb. Multisensory Process., pp. 775-792, 2012.

[8] M. V. Sanchez-Vives, B. Spanlang, A. Frisoli, M. Bergamasco, and M. Slater, "Virtual hand illusion induced by visuomotor correlations," PLoS One, vol. 5, no. 4, pp. 1-6, 2010.

[9] D. Cowie, A. McKenna, A. J. Bremner, and J. E. Aspell, "The development of bodily self-consciousness: changing responses to the Full Body Illusion in childhood," Dev. Sci., vol. 21, no. 3, pp. 1-12, 2018.

[10] J. O. Bailey and J. N. Bailenson, "Considering virtual reality in children's lives," Journal of Children and Media, vol. 11, no. 1. Routledge, pp. 107-113, Jan-2017.

[11] J. O. Bailey and J. N. Bailenson, "Immersive Virtual Reality and the Developing Child," in Cognitive Development in Digital Contexts, Elsevier Inc., 2017, pp. 181-200.

[12] S. R. Department, "Statistica - Active virtual reality users worldwide," 2020.2 [Online]. Available: www.statista.com/statistics/426469/active-virtual-realityusers-worldwide/.

[13] S. J. G. Ahn, J. Bostick, E. Ogle, K. L. Nowak, K. T. McGillicuddy, and J. N. Bailenson, “Experiencing Nature: Embodying Animals 
in Immersive Virtual Environments Increases Inclusion of Nature in Self and Involvement With Nature," J. Comput. Commun., vol. 21, no. 6, pp. 399-419, 2016.

[14] L. Maister, M. Slater, M. V. Sanchez-Vives, and M. Tsakiris, "Changing bodies changes minds: Owning another body affects social cognition," Trends Cogn. Sci., vol. 19, no. 1, pp. 6-12, 2015.

[15] T. C. Peck, S. Seinfeld, S. M. Aglioti, and M. Slater, "Putting yourself in the skin of a black avatar reduces implicit racial bias," Conscious. Cogn., vol. 22, no. 3, pp. 779-787, 2013.

[16] A. S. Won, J. Bailenson, J. Lee, and J. Lanier, "Homuncular Flexibility in Virtual Reality," J. Comput. Commun., vol. 20, no. 3, pp. 241-259, 2015.

[17] K. Ma and B. Hommel, "Body-ownership for actively operated non-corporeal objects," Conscious. Cogn., vol. 36, pp. 75-86, 2015.

[18] A. M. de Haan, M. Smit, S. Van der Stigchel, S. A. Keyner, and H. C. Dijkerman, "Body representation does not lag behind in updating for the pubertal growth spurt," J. Exp. Child Psychol., vol. 175 , pp. 48-66, 2018.

[19] J. O. Bailey, J. N. Bailenson, J. Obradovi, and N. R. Aguiar, "Virtual reality's effect on children's inhibitory control, social compliance, and sharing," J. Appl. Dev. Psychol., vol. 64, Jul. 2019.

[20] L. E. Miller, A. Cawley-Bennett, M. R. Longo, and A. P. Saygin, "The recalibration of tactile perception during tool use is body-part specific," Exp. Brain Res., vol. 235, no. 10, pp. 29172926, 2017.

[21] D. A. Bowman and L. F. Hodges, "An Evaluation of Techniques for Grabbing and Manipulating Remote Objects in Immersive Virtual Environments," Proc. 1997 Symp. Interact. 3D Graph., pp. 35-ff, 1997.

[22] L. B. Smith, C. Yu, and A. F. Pereira, "Not your mother's view: the dynamics of toddler visual experience," Dev. Sci., vol. 14, no. 1, pp. 9-17, 2011.

[23] D. Cowie, T. R. Makin, and A. J. Bremner, “Children's Responses to the Rubber-Hand Illusion Reveal Dissociable Pathways in Body Representation," Psychol. Sci., vol. 24, no. 5, pp. 762-769, 2013.

[24] D. Corbetta and W. Snapp-Childs, "Seeing and touching: The role of sensory-motor experience on the development of infant reaching," Infant Behav. Dev., vol. 32, pp. 44-58, 2009.

[25] E. Thelen and L. B. Smith, A dynamic systems approach to the development of cognition and action. MA: MIT press, 1996.

[26] C. K. Rovee and D. T. Rovee, "Conjugate reinforcement of infant exploratory behavior," J. Exp. Child Psychol., vol. 8, no. 1, pp. 33-39, 1969.

[27] J. T. Delafield-Butt, Y. Freer, J. Perkins, D. Skulina, B. Schögler, and D. N. Lee, "Prospective organization of neonatal arm movements: A motor foundation of embodied agency, disrupted in premature birth," Dev. Sci., vol. 21, no. 6, pp. 1-16, 2018.

[28] S. Zoia et al., "Evidence of early development of action planning in the human foetus: A kinematic study," Exp. Brain Res., vol. 176, no. 2, pp. 217-226, 2007.

[29] L. E. Bahrick and J. S. Watson, "Detection of Intermodal Proprioceptive-Visual Contingency as a Potential Basis of SelfPerception in Infancy," Dev. Psychol., vol. 21, no. 6, pp. 963-973, 1985.

[30] R. Morgan and P. Rochat, "Intermodal calibration of the body in early infancy," Ecol. Psychol., vol. 9, no. 1, pp. 1-23, 1997.

[31] P. Rochat, "Self-perception and action in infancy," Exp. Brain
Res., vol. 123, no. 1-2, pp. 102-109, 1998.

[32] M. L. Filippetti, M. H. Johnson, S. Lloyd-Fox, D. Dragovic, and T. Farroni, "Body perception in newborns," Curr. Biol., vol. 23, no. 23, pp. 2413-2416, 2013.

[33] N. Zmyj, J. Jank, S. Schütz-Bosbach, and M. M. Daum, “Detection of visual-tactile contingency in the first year after birth," Cognition, vol. 120, no. 1, pp. 82-89, 2011.

[34] L. E. Bahrick, "Body perception: Intersensory origins of self and other perception in newborns," Curr. Biol., vol. 23, no. 23, pp. R1039-R1041, 2013.

[35] A. J. Bremner and D. Cowie, "Developmental origins of the hand in the mind, and the role of the hands in the development of the mind," in Z. Radman (Ed.), The hand: An organ of the mind. What the manual tells the mental, vol. (Ed), MIT Press: Cambridge, MA, 2013, pp. 27-55.

[36] M. Botvinick and J. Cohen, "Rubber hands 'feel' touch that eyes see," Nature, vol. 391, p. 756, 1998.

[37] M. Tsakiris and P. Haggard, "The Rubber Hand Illusion Revisited: Visuotactile Integration and Self-Attribution," J. Exp. Psychol. Hum. Percept. Perform., vol. 31, no. 1, pp. 80-91, 2005.

[38] D. Cowie, S. Sterling, and A. J. Bremner, "The development of multisensory body representation and awareness continues to 10years of age: Evidence from the rubber hand illusion," J. Exp. Child Psychol., vol. 142, pp. 230-238, 2016.

[39] E. Nava, N. Bolognini, and C. Turati, "The Development of a Cross-Modal Sense of Body Ownership," Psychol. Sci., vol. 28, pp. 330-337, 2017.

[40] M. L. Filippetti and L. Crucianelli, "If I were a grown-up: Children's response to the rubber hand illusion with different hand sizes," J. Exp. Child Psychol., vol. 185, pp. 191-205, 2019.

[41] J. J. Braithwaite, D. G. Watson, and H. Dewe, "Predisposition to Out-of-Body Experience (OBE) is Associated with Aberrations in Multisensory Integration: Psychophysiological Support from a \&quot; Rubber-hand Illusion \&quot; Study," vol. 43, no. 6, pp. 1125-1143, 2017.

[42] M. Tsakiris, G. Prabhu, and P. Haggard, "Having a body versus moving your body: How agency structures body-ownership," Conscious. Cogn., vol. 15, no. 2, pp. 423-432, 2006.

[43] M. R. Longo and P. Haggard, "Sense of agency primes manual motor responses," Perception, vol. 38, no. 1, pp. 69-78, 2009.

[44] M. Tsakiris, M. R. Longo, and P. Haggard, "Having a body versus moving your body: Neural signatures of agency and bodyownership," Neuropsychologia, vol. 48, no. 9, pp. 2740-2749, 2010.

[45] M. V. Sanchez-Vives and M. Slater, "Opinion: From presence to consciousness through virtual reality," Nat. Rev. Neurosci., vol. 6, no. 4, pp. 332-339, 2005.

[46] M. Slater, D. Perez-Marcos, H. H. Ehrsson, and M. V. SanchezVives, "Towards a digital body: The virtual arm illusion," Front. Hum. Neurosci., vol. 2, no. August, pp. 1-8, 2008.

[47] A. Maselli and M. Slater, "Sliding perspectives: dissociating ownership from self-location during full body illusions in virtual reality," Front. Hum. Neurosci., vol. 8, no. September, pp. 1-19, 2014.

[48] V. I. Petkova and H. H. Ehrsson, "If I Were You: Perceptual Illusion of Body Swapping," PLoS One, vol. 3, no. 12, p. e3832, 2008.

[49] M. Slater, B. Spanlang, M. V. Sanchez-Vives, and O. Blanke, "First person experience of body transfer in virtual reality," PLoS One, vol. 5 , no. 5, pp. 1-9, 2010. 
[50] M. Slater et al., "An experimental study of a virtual reality counselling paradigm using embodied self-dialogue," Sci. Rep., vol. 9, no. 1, pp. 1-13, 2019.

[51] D. Banakou, R. Groten, and M. Slater, "Illusory ownership of a virtual child body causes overestimation of object sizes and implicit attitude changes," Proc. Natl. Acad. Sci. U.S. A., vol. 110, no. 31, pp. 12846-12851, 2013.

[52] I. V. Piryankova et al., "Owning an overweight or underweight body: Distinguishing the physical, experienced and virtual body," PLoS One, vol. 9, no. 8, 2014.

[53] L. Hoyet, F. Argelaguet, C. Nicole, and A. Lécuyer, "'Wow! i have six fingers!': Would you accept structural changes of your hand in VR?," Front. Robot. AI, vol. 3, no. MAY, pp. 1-12, 2016.

[54] K. Kilteni, J. M. Normand, M. V. Sanchez-Vives, and M. Slater "Extending body space in immersive virtual reality: A very long arm illusion," PLoS One, vol. 7, no. 7, 2012.

[55] L. Lin and S. Jörg, "Need a hand? How appearance affects the virtual hand illusion," Proc. ACM Symp. Appl. Perception, SAP 2016, pp. 69-76, 2016.

[56] L. Aymerich-Franch, D. Petit, G. Ganesh, and A. Kheddar, "Nonhuman Looking Robot Arms Induce Illusion of Embodiment," Int. J. Soc. Robot., vol. 9, no. 4, pp. 479-490, 2017.

[57] W. Steptoe, A. Steed, and M. Slater, "Human tails: Ownership and control of extended humanoid avatars," IEEE Trans. Vis. Comput. Graph., vol. 19, no. 4, pp. 583-590, 2013.

[58] J. M. Gottwald et al., "The Developing Bodily Self: How Posture Constrains Body Representation in Childhood," Child Dev., vol. 92, no. 1, pp. 351-366, 2021.

[59] S. Keenaghan, M. Polaskova, S. Thurlbeck, R. W. Kentridge, and D. Cowie, "Alice in Wonderland: The effects of body size and movement on children's size perception and bodily awareness in virtual reality," (preprint), no. March 16, 2020.

[60] D. Freeman et al., "Automated psychological therapy using immersive virtual reality for treatment of fear of heights: a single-blind, parallel-group, randomised controlled trial," The Lancet Psychiatry, vol. 5, no. 8, pp. 625-632, 2018.

[61] M. Roussou, "A VR Playground for Learning Abstract Mathematics Concepts," IEEE Comput. Graph. Appl., vol. 29, no. 1, pp. 82-85, Jan. 2009.

[62] H. G. Hoffman et al., "Immersive Virtual Reality as an Adjunctive Non-opioid Analgesic for Pre-dominantly Latin American Children With Large Severe Burn Wounds During Burn Wound Cleaning in the Intensive Care Unit: A Pilot Study," Front. Hum. Neurosci., vol. 13, p. 262, Aug. 2019.

[63] H. Adams, G. Narasimham, J. Rieser, S. Creem-Regehr, J. Stefanucci, and B. Bodenheimer, "Locomotive recalibration and prism adaptation of children and teens in immersive virtual environments," IEEE Trans. Vis. Comput. Graph., vol. 24, no. 4, pp. 1408-1417, 2018.

[64] B. A. Morrongiello, M. Corbett, M. Milanovic, and J. Beer, “Using a Virtual Environment to Examine How Children Cross Streets: Advancing Our Understanding of How Injury Risk Arises," J. Pediatr. Psychol., vol. 41, no. 2, pp. 265-275, 2016.

[65] S. V. Babu et al., "An immersive virtual peer for studying social influences on child cyclists' road-crossing behavior," IEEE Trans. Vis. Comput. Graph., vol. 17, no. 1, pp. 14-25, 2011.

[66] N. Yee and J. Bailenson, "The proteus effect: The effect of transformed self-representation on behavior," Hum. Commun. Res., vol. 33, no. 3, pp. 271-290, 2007.

[67] K. Y. Segovia and J. N. Bailenson, “Virtually True: Children's
Acquisition of False Memories in Virtual Reality," Media Psychol., vol. 12, no. 4, pp. 371-393, Nov. 2009.

[68] D. Bates, M. Mächler, B. M. Bolker, and S. C. Walker, "Fitting linear mixed-effects models using lme4," in Journal of Statistical Software, vol. 67, no. 1, 2015.

[69] R. C. Team, "The R Project for Statistical Computing," Http://Www.R-Project.Org/,pp. 1-12, 2013.

[70] H. Wickham, "ggplot2: elegant graphics for data analysis." Springer New York, 2009.

[71] Y. Benjamini and Y. Hochberg, "Controlling the False Discovery Rate: a Practical and Powerful Approach to Multiple Testing," J. R. Stat. Soc. Ser. B Stat. Methodol., vol. 57, no. 1, pp. 289-300, 2009.

[72] B. A. Schneider, M. Avivi-reich, and M. Mozuraitis, "A cautionary note on the use of the Analysis of Covariance ( ANCOVA ) in classification designs with and without withinsubject factors," vol. 6, no. April, pp. 1-12, 2015.

[73] H. H. Ehrsson, "The experimental induction of out-of-body experiences," Science (80-. )., vol. 317, no. 5841, p. 1048, 2007.

[74] M. Slater, D. Perez-Marcos, H. H. Ehrsson, and M. V. SanchezVives, "Inducing illusory ownership of a virtual body," Front. Neurosci., vol. 3, no. SEP, pp. 214-220, 2009.

[75] J. J. Braithwaite, E. Broglia, and D. G. Watson, "Autonomic emotional responses to the induction of the rubber-hand illusion in those that report anomalous bodily experiences: Evidence for specific psychophysiological components associated with illusory body representations," J. Exp. Psychol. Hum. Percept. Perform., vol. 40, no. 3, pp. 1131-1145, 2014.

[76] Y. Yuan and A. Steed, "Is the Rubber Hand Illusion Induced by Immersive Virtual Reality?," in IEEE Virtual Reality Conference (VR) IEEE, 2010, pp. 95-102.

[77] M. Tsakiris, L. Carpenter, D. James, and A. Fotopoulou, "Hands only illusion: Multisensory integration elicits sense of ownership for body parts but not for non-corporeal objects," Exp. Brain Res., vol. 204, no. 3, pp. 343-352, 2010.

[78] A. K. Seth, K. Suzuki, and H. D. Critchley, "An interoceptive predictive coding model of conscious presence," Front. Psychol., vol. 2, pp. 1-16, 2012.

[79] B. Lenggenhager, T. Tadi, T. Metzinger, and O. Blanke, "Video ergo sum: Manipulating bodily self-consciousness," Science (80.)., vol. 317, no. 5841, pp. 1096-1099, 2007.

[80] A. Haans, W. A. IJsselsteijn, and Y. A. W. de Kort, "The effect of similarities in skin texture and hand shape on perceived ownership of a fake limb," Body Image, vol. 5, no. 4, pp. 389-394, 2008.

[81] J. O. Wobbrock, L. Findlater, D. Gergle, and J. J. Higgins, "The Aligned Rank Transform for Nonparametric Factorial Analyses Using Only ANOVA Procedures," Proc. SIGCHI Conf. Hum. factors Comput. Syst., pp. 143-146, 2011.

[82] K. C. Armel and V. S. Ramachandran, "Projecting sensations to external objects: Evidence from skin conductance response," Proc. R. Soc. B Biol. Sci., vol. 270, no. 1523, pp. 1499-1506, 2003.

[83] M. Rohde, M. Luca, and M. O. Ernst, "The rubber hand illusion: Feeling of ownership and proprioceptive drift Do not go hand in hand," PLoS One, vol. 6, no. 6, pp. 1-8, 2011

[84] A. Kalckert and H. H. Ehrsson, "Moving a Rubber Hand that Feels Like Your Own: A Dissociation of Ownership and Agency," Front. Hum. Neurosci., vol. 6, no. March, pp. 1-14, 2012.

[85] T. C. Peck, L. E. Sockol, and S. M. Hancock, "Mind the Gap: The Underrepresentation of Female Participants and Authors in 
Virtual Reality Research," IEEE Trans. Vis. Comput. Graph., vol. 26, no. 5, pp. 1945-1954, 2020.

[86] P. Litwin, B. Zybura, and P. Motyka, "Tactile information counteracts the attenuation of rubber hand illusion attributable to increased visuo-proprioceptive divergence," PLoS One, vol. 15, no. 12 December, pp. 1-20, 2020.

[87] M. Gonzalez-Franco and T. C. Peck, "Avatar embodiment. Towards a standardized questionnaire," Front. Robot. AI, vol. 5, no. JUN, pp. 1-9, 2018.

[88] T. C. Peck and M. Gonzalez-Franco, "Avatar embodiment. A standardized questionnaire," Front. Virtual Real., vol. 1, p. 575943, 2021.

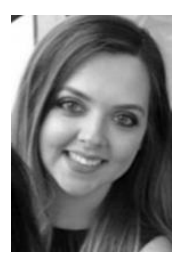

Hayley Dewe obtained a BSc (2012) and MRes (2013) in Psychology at the University of Birmingham, United Kingdom. She worked as a Research Assistant (201315) and Research Fellow (2015-16) at the University of Birmingham, United Kingdom, where she was awarded her PhD in 2018. Since 2019, she has been working as a Postdoctoral Research Fellow in the Department of Psychology at Durham University, United Kingdom. She is a member of the British Neuropsychological Society, has 6 peer-reviewed publications and has written several articles and press releases as part of wider scientific engagement. Her research interests include body representation, consciousness, and sensorimotor development.

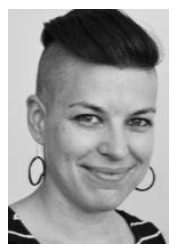

Janna Gottwald received a Dipl.-Psych. Psychology diploma $(\approx \mathrm{MSc})$ at the University of Potsdam, Germany (2011) and a PhD in Psychology at Uppsala University, Sweden (2016). She worked as a Postdoctoral Research Associate at the International PsychoanaIytic University Berlin, Germany (2016-17) and at Durham University, United Kingdom (2018). She is Senior Researcher at the Uppsala Child \& Baby Lab at the Psychology Department of Uppsala University and PI on a grant from the Swedish Research Council (2018-20, 3.3M SEK): 'An Embodied Account of Executive Function Development'. She received the Outstanding Young Researcher in Psychology Award from the Swedish National Committee for Psychological Sciences (2019) and a Benzelius Prize from the Swedish Royal Society of Sciences (2018). Her interests are embodied cognitive science, sensorimotor and cognitive development from infancy to childhood.

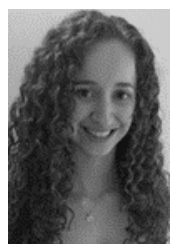

Laura-Ashleigh Bird gained a BSc in Psychology (2015) and MSc in Developmental Disorders (2016) at Lancaster University, United Kingdom. She was a Research Associate at Lancaster University (2016-17) and Research Assistant at Durham University, United Kingdom (2017-19). She is currently a Research Assistant at University College London, United Kingdom and an honorary associate at Durham University. She has 5 peer-reviewed publications, with interests in motor development, particularly upper limb differences.

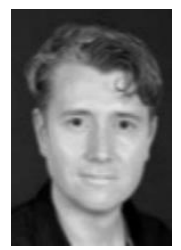

Harry Brenton has spent 15 years researching and developing the use of Al for social VR. He has applied these technologies for clients such as the NHS, Imperial College London, Harvard Medical School, Deloitte and Alchemy VR. He has published widely on immersive technologies and lectures at NYU in immersive storytelling. He has a PhD in VR and medical simulation from Imperial College London, United Kingdom and is CEO and founder of BespokeVR.com.

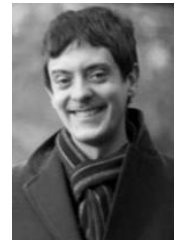

Marco Gillies completed his BA (1997) and PhD in Computer Science (2001) at Cambridge University, United Kingdom. He worked as a Postdoctoral Researcher in Computer Science at UCL, United Kingdom (2001-08). Currently, he is a reader in Computing and now Academic Director of Distance Learning at Goldsmiths, University of London, United Kingdom. He is co-founder of the Embodied Audio-Visual Interaction (EAVI) group (Goldsmiths) and has had funded research projects from the EPSRC, NESTA and the European Commission. With over 50 peer reviewed publications, his research interests include Virtual reality, Interactive Machine Learning, Motion Capture, Animated Virtual Characters, Arts Computing.

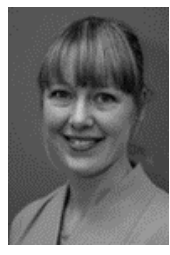

Dorothy Cowie received a BA (2003) and PhD (2007) in Experimental Psychology at the University of Oxford, United Kingdom. She was a Postdoctoral Fellow at UCL Institute of Neurology, United Kingdom (200710) and a Lecturer at Goldsmiths Psychology Department, United Kingdom (2010-13). She is currently an Associate Professor in Psychology at Durham University, UK, where she holds an ESRC grant: 'The Development of Own-Body Representation in Childhood' (2017, £500k). She is editor for Multisensory Research (since 2018) and review editor for Frontiers in Psychology (Developmental, since 2019). She has 26 peer-reviewed publications, and her research interests include the development of own-body representation and visuomotor control. 